Computational Physics and Engineering Division (10)

\title{
Computational Experience with the Reich-Moore Resolved-Resonance Equations in the AMPX Cross-Section Processing System
}

\author{
M. E. Dunn \\ Oak Ridge National Laboratory, \\ P. O. Box 2008, \\ Oak Ridge, TN 37831-6370, USA \\ Submitted to the \\ 2001 ANS International Meeting on Mathematical \\ Methods for Nuclear Applications, \\ September 9-13, 2001, \\ Little America Hotel, \\ Salt Lake City, Utah, USA \\ Organized and Sponsored by the \\ Mathematics \& Computation Division and the Idaho Section of ANS \\ Co-sponsored by the \\ Reactor Physics Division of ANS and the \\ OECD/Nuclear Energy Agency
}

The submitted manuscript has been authored by a contractor of the U.S. Government under contract No. DE-AC05-00OR22725. Accordingly, the U.S. Government retains a nonexclusive, royalty-free license to publish or reproduce the published form of this contribution, or allow others to do so, for U.S. Government purposes.

*Managed by UT-Battelle, LLC, under contract DE-AC05-00OR22725 with the U.S. Department of Energy. 


\title{
COMPUTATIONAL EXPERIENCE WITH THE REICH-MOORE RESOLVED-RESONANCE EQUATIONS IN THE AMPX CROSS-SECTION PROCESSING SYSTEM
}

\author{
M. E. Dunn \\ Oak Ridge National Laboratory, \\ P. O. Box 2008, \\ Oak Ridge, TN 37831-6370, USA \\ dunnme@ornl.gov
}

Keywords: Reich-Moore, resolved-resonance region, cross sections

\begin{abstract}
The Reich-Moore formulation is used extensively in many isotope/nuclide evaluations to represent neutron cross section data for the resolved-resonance region. The Reich-Moore equations require the evaluation of complex matrices (i.e., matrices with complex quantities) that are a function of the resonance energy and corresponding resonance parameters. Although the Reich-Moore equations are documented in the open literature, computational pitfalls may be encountered with the implementation of the Reich-Moore equations in a cross-section processing code. Based on experience, numerical instabilities in the form of nonphysical oscillations can occur in the calculated absorption, capture or elastic scattering cross sections. To illustrate possible numerical instabilities, the conventional Reich-Moore equations are presented, and the conditions that lead to numerical problems in the cross-section calculations are identified and demonstrated for ${ }^{28} \mathrm{Si}$ and ${ }^{60} \mathrm{Ni}$. In an effort to circumvent the computational problems, detailed or revised Reich-Moore expressions have been developed to efficiently and accurately calculate cross sections for neutron-induced reactions in the resolved-resonance region. The revised equations can be used to avoid numerical problems associated with the implementation of the Reich-Moore formulation in a cross-section processing code. The revised Reich-Moore equations are also used to demonstrate the improved cross-section results (i.e., without numerical instabilities) for ${ }^{28} \mathrm{Si}$ and ${ }^{60} \mathrm{Ni}$.
\end{abstract}

\section{INTRODUCTION}

Radiation-transport calculations require the use of energy-dependent cross-section data to solve the Boltzmann transport equation for fissile systems. For neutron-induced reactions, the Evaluated Nuclear Data File (ENDF) system has procedures and formats that are used to describe the complex structure of cross-section data for specific isotopes/nuclides of interest (ENDF/102, 1991). In a cross-section evaluation for a particular material that is characterized by neutron resonances, a resonance formalism (e.g., Reich-Moore, single-levelBreit Wigner, etc.) is specified to reconstruct the energy-dependent cross-section function in the resonance region. For the specified resonance formalism, experimentally determined resonance parameters (e.g., resonance energy, partial widths, etc.) are also provided in the cross-section evaluation. Since radiation transport codes do not directly access the data in a cross-section evaluation, a processing code 
must be used to read the parameters and reconstruct the energy-dependent cross-section function that is to be used in a transport calculation. One of the most widely used resonance representations in ENDF/B-6 is the Reich-Moore (RM) formalism. Although the RM equations are well documented in the open literature, the implementation of the RM equations can lead to numerical instabilities in the calculation of absorption, capture and elastic scattering cross sections under certain conditions. The objective of this paper is to present the RM formulae and identify the conditions that lead to numerical instabilities in the cross-section calculations. Moreover, the paper provides detailed expressions that can be used to avoid numerical problems in calculating cross sections in the resolved-resonance region using the RM representation.

\section{REICH-MOORE RESONANCE EQUATIONS}

Resonance representations are essentially interaction models that describe the interaction between a neutron and target nucleus. Because of the complexity of the internal structure of a nucleus, the resonance representations do not model the nuclear effects within the nucleus. However, the resonance parameters that are provided in a cross-section evaluation are strongly correlated to the internal properties of a nucleus and are obtained by the evaluation of measured cross-section data. With regard to the RM representation, the RM formalism is an approximation to the general R-matrix theory for describing neutron-nucleus interactions. Since the RM formulae are documented in the open literature, a derviation of the RM formulae is not provided in the subsequent discussion; rather, the defining equations of the approximation are presented. In the RM approximation, the off-diagonal contribution to the R-matrix from the photon channels is neglected. The resulting formalism is especially suited for representing isotopes that are characterized with many resonances. Although the ENDF formulation of the RM formulae restricts the number of fission channels to two, the ENDF formulation is well suited for fissile isotopes because the fission process occurs through a small number of channels. For neutroninduced reactions, the general expression for a cross section as a function of energy with an exit channel $c$ is given by the following equation (ENDF-102, 1991):

$$
\sigma_{n c}(E)=\frac{\pi}{k^{2}} \sum_{J} g_{J}\left|\delta_{n c}-U_{n c}^{J}\right|^{2},
$$

Note that the subscripts $n$ and $c$ in Eq. (1) correspond to entrance and exit channels, respectively, and the angular momentum subscripts, $\ell$, are implied. In the subsequent equations, the angular momentum subscripts are also implied. In Eq. (1), the exit channel $c$ can be scattering $(n)$, capture $(\gamma)$ or either of the two partial-fission widths $\left(f_{l}\right.$ or $\left.f_{2}\right)$. The collision matrix is a complex matrix that is defined as follows:

$$
U_{n c}^{J}=e^{-i\left(\phi_{n}+\phi_{c}\right)}\left[2((I-K))_{n c}^{-1}-\delta_{n c}\right],
$$

where 


$$
(I-K)_{n c}=\delta_{n c}-\frac{i}{2} \sum_{r} \frac{\Gamma_{n r}^{1 / 2} \Gamma_{c r}^{1 / 2}}{\left(E_{r}-E\right)-i \frac{\Gamma_{\gamma r}}{2}}
$$

The total and elastic scattering cross sections can be expressed as a function of energy in terms of the collision matrix:

$$
\sigma_{t}(E)=\frac{2 \pi}{k^{2}} \sum_{J} g_{J} \operatorname{Re}\left(1-U_{n n}^{J}\right)
$$

and

$$
\sigma_{n, n}(E)=\frac{\pi}{k^{2}} \sum_{J} g_{J}\left|1-U_{n n}^{J}\right|^{2}
$$

Likewise, the fission cross section can be calculated in terms of the collision matrix and corresponding exiting fission channels:

$$
\sigma_{f}(E)=\frac{\pi}{k^{2}} \sum_{J} g_{J} \sum_{c}\left|U_{n c}^{J}\right|^{2}
$$

For the subsequent discussion, a matrix $\rho$ can be defined as follows:

$$
\rho_{n c}=\left(I-(I-K)^{-1}\right)_{n c}=-\left((I-K)^{-1} K\right)_{n c} .
$$

Using Eq. (7), the collision matrix can be expressed in the following format:

$$
U_{n c}^{J}=e^{-i\left(\phi_{n}+\phi_{c}\right)}[2(I-\rho)-I]_{n c}=e^{-i\left(\phi_{n}+\phi_{c}\right)}[I-2 \rho]_{n c} .
$$

An expression for the elastic scattering cross section is obtained by expanding the squared quantity in Eq. (5) and substituting the expression from Eq. (8) for the collision matrix into the expanded form of Eq. (5). After simplification, the following expression is obtained for the elastic scattering cross section:

$$
\sigma_{e l}(E)=\sigma_{1,1}(E)=\frac{4 \pi}{k^{2}} \sum_{J} g_{J}\left\{-2 \sin ^{2} \phi_{1} \operatorname{Re} \rho_{11}+\sin \left(2 \phi_{1}\right) \operatorname{Im} \rho_{11}+\left|\rho_{11}\right|^{2}+\sin ^{2} \phi_{1}\right\} .
$$

In Eq. (9), the neutron subscript $(n)$ is replaced with the value of 1 for the purposes of discussion. Likewise, in Eq. (9), the phase-shift subscripts correspond to the channel number while the angular- momentum subscripts are implied. Also, note that Eq. (9) includes an implicit summation over the channel spin and resonance spin $J$ for the potential scattering contribution. Additional details for calculating the potential scattering contribution are provided by Dunn and Greene (2000). 
Using a similar procedure as for the elastic scattering cross section, an expression is obtained for the total cross section by substituting the expression for the collision matrix into Eq. (4). Upon further simplification the following expression is obtained for the total cross section:

$$
\sigma_{t}(E)=\frac{4 \pi}{k^{2}} \sum_{J} g_{J}\left\{\left(1-2 \sin ^{2} \phi_{1}\right) \operatorname{Re} \rho_{11}+\sin \left(2 \phi_{1}\right) \operatorname{Im} \rho_{11}+\sin ^{2} \phi_{1}\right\} .
$$

An expression for the fission cross section is obtained by expanding the squared quantity in Eq. (6) and inserting the expression for the collision matrix from Eq. (8) into Eq. (6). The resulting equation for the fission cross section has the following form:

$$
\sigma_{f}(E)=\frac{4 \pi}{k^{2}} \sum_{J} g_{J}\left\{\left|\rho_{12}\right|^{2}+\left|\rho_{13}\right|^{2}\right\} .
$$

In Eq. (11), the first- and second-fission channels are denoted with the values 2 and 3, respectively. By subtracting Eq. (9) from Eq. (10), an expression is also obtained for the absorption cross section as a function of energy:

$$
\sigma_{a}(E)=\frac{4 \pi}{k^{2}} \sum_{J} g_{J}\left\{\operatorname{Re} \rho_{11}-\left|\rho_{11}\right|^{2}\right\} .
$$

Likewise, an equation for the capture cross section as a function of energy is obtained by subtracting Eq. (11) from Eq. (12):

$$
\sigma_{\gamma}(E)=\frac{4 \pi}{k^{2}} \sum_{J} g_{J}\left\{\operatorname{Re} \rho_{11}-\left|\rho_{11}\right|^{2}-\left|\rho_{12}\right|^{2}-\left|\rho_{13}\right|^{2}\right\} .
$$

Eqs. (9) through (13) are the Reich-Moore representations for the elastic scattering, total, fission, absorption and capture cross sections, respectively. In the following section, the numerical aspects of the RM equations are explored in greater detail.

\section{COMPUTATIONAL CONSIDERATIONS}

\subsection{Absorption and Capture}

The Reich-Moore equations that are presented in Section 2 are a function of the complex matrix $\rho$. The formulation of $\rho$ as presented in Eq. (7) was initially proposed by Larson (1993) to circumvent numerical instabilities that can occur when the evaluation of $\rho$ involves small differences of large numbers. Nonphysical oscillations may occur during the calculation of lowenergy capture cross sections for some isotopes, and the reformulation of $\rho$ as presented in Eq. (7) was developed to avoid the observed oscillations in the capture cross section. Despite the reformulation for $\rho$ in Eq. (7), unexpected numerical problems can still occur depending upon the implementation of the absorption, capture or elastic scattering cross section formulae. In this section, the conditions that lead to numerical problems in the absorption and capture cross section 
are discussed, and in Section 3.2, conditions that lead to numerical problems with the elastic scattering cross section are also presented.

As shown in Eq. (12), the absorption cross section calculation involves the computation of the quantity $R e \rho_{11}-\left|\rho_{11}\right|^{2}$. At ORNL, the AMPX module POLIDENT (Dunn and Greene, 2000), which generates continuous energy cross sections from ENDF data, has been used to process more than 300 ENDF/B-6 evaluations. Experience with the cross-section calculations has revealed that a numerical instability can occur for isotopes that have a very small absorption or capture cross section (e.g., $\sim 10^{-5}$ ) coupled with a detailed resonance structure in the absorption or capture cross section. Since $\rho_{11}$ is a complex quantity, $\rho_{11}$ has the form a $+i b$. Consequently, the quantity $\left|\rho_{11}\right|^{2}$ is equal to $a^{2}+b^{2}$. If $a$ and $b$ are less than 1 and the quantity $b$ is approximately equal to $\mathrm{a}^{1 / 2}$, the difference calculation in Eq. (12) involves the subtraction of two small numbers that are approximately equal. If the calculation in Eq. (12) is performed using the doubleprecision complex functions that are available in FORTRAN on a very fine energy grid, numerical instabilities occur for isotopes/nuclides with very small absorption cross-section values. Therefore, an alternative approach must be used to accurately calculate the absorption cross section.

A similar numerical instability problem can occur in the capture cross-section calculation using Eq. (13) with the complex functions that are available in FORTRAN. To avoid the instability problems, the quantity within braces in Eq. (13) must be re-cast into a different form to accurately calculate the capture cross section. In the subsequent discussion, alternate forms of Eqs. (12) and (13) are developed, and calculational results are presented using both the original and new cross-section formulations. Although numerical problems are possible with Eqs. (12) and (13), no problems have been observed in the fission cross section as defined by Eq. (11) which requires the addition of $\left|\rho_{12}\right|^{2}$ and $\left|\rho_{13}\right|^{2}$. Therefore, the fission cross section is not included in the following discussion.

As noted above, the calculation of the absorption cross section requires the evaluation of the following quantity:

$$
\operatorname{Re} \rho_{11}-\left|\rho_{11}\right|^{2}
$$

Likewise, the capture cross section requires the computation of the following quantity:

$$
\operatorname{Re} \rho_{11}-\left|\rho_{11}\right|^{2}-\left|\rho_{12}\right|^{2}-\left|\rho_{13}\right|^{2} .
$$

Because $n$ and $c$ represent the entrance and exit channels, respectively, in Eq. (7), the quantity $\rho_{n c}$ is a $n \times c$ matrix, and for neutron-induced reactions in the ENDF formulation of the Reich-Moore equations, $\rho_{n c}$ is a $3 \times 3$ matrix. To facilitate the development, the matrix quantity $(I-K)^{-1}$ can be defined as $A$, and $\rho_{n c}$ is calculated by multiplying $A$ and $K$. In order to evaluate the expressions in Eqs. (14) and (15), the quantities $\rho_{11}, \rho_{12}$ and $\rho_{13}$ can be calculated by 
multiplying $A$ and the symmetric matrix $K$, and the following expressions are obtained for $\rho_{11}, \rho_{12}$ and $\rho_{13}$ :

$$
\begin{aligned}
& -\rho_{11}=A_{11} K_{11}+A_{12} K_{12}+A_{13} K_{13}, \\
& -\rho_{12}=A_{11} K_{12}+A_{12} K_{22}+A_{13} K_{23}, \\
& -\rho_{13}=A_{11} K_{13}+A_{12} K_{23}+A_{13} K_{33} .
\end{aligned}
$$

The individual elements of the $K$ matrix can be obtained from Eq. (3); however, the elements of the $A$ matrix require some additional effort. In order to evaluate the $A$ matrix, the matrix quantity $(I-K)$ that is defined by Eq. (3) can be defined as $B$ which is a $3 \times 3$ matrix. Since $A$ is the inverse of $B$, the product of $A$ and $B$ is the identity matrix, and a set of nine equations with nine unknowns is obtained by the multiplication of $A$ and $B$. After some algebraic simplification, the following equations are obtained for the quantities $A_{11}, A_{12}$ and $A_{13}$ :

$$
\begin{aligned}
& A_{11}=\frac{\left(1-K_{22}\right)\left(1-K_{33}\right)-K_{23}^{2}}{D E N}, \\
& A_{12}=\frac{K_{13} K_{23}+K_{12}\left(1-K_{33}\right)}{D E N}, \\
& A_{13}=\frac{K_{12} K_{23}+K_{13}\left(1-K_{22}\right)}{D E N},
\end{aligned}
$$

where

$$
\begin{aligned}
D E N= & \left(1-K_{11}\right)\left(1-K_{22}\right)\left(1-K_{33}\right)-2 K_{12} K_{13} K_{23} \\
& -K_{23}^{2}\left(1-K_{11}\right)-K_{12}^{2}\left(1-K_{33}\right)-K_{13}^{2}\left(1-K_{22}\right) .
\end{aligned}
$$

The elements of the $K$ matrix are defined in Table 1. 
Table 1 Definitions of the elements of the $K$ matrix $^{\dagger}$

$$
\begin{array}{lll}
K_{11}=-\frac{1}{2}\left(B^{\prime}-i A^{\prime}\right) & A^{\prime}=\sum_{r=1}^{N R} \frac{\Gamma_{1 r} a}{a^{2}+b^{2}} & B^{\prime}=\sum_{r=1}^{N R} \frac{\Gamma_{1 r} b}{a^{2}+b^{2}} \\
K_{12}=-\frac{1}{2}(D-i C) & C=\sum_{r=1}^{N R} \frac{\sqrt{\Gamma_{1 r}} \sqrt{\Gamma_{2 r}} a}{a^{2}+b^{2}} & D=\sum_{r=1}^{N R} \frac{\sqrt{\Gamma_{1 r}} \sqrt{\Gamma_{2 r}} b}{a^{2}+b^{2}} \\
K_{13}=-\frac{1}{2}(F-i E) & E=\sum_{r=1}^{N R} \frac{\sqrt{\Gamma_{1 r}} \sqrt{\Gamma_{3 r}} a}{a^{2}+b^{2}} & F=\sum_{r=1}^{N R} \frac{\sqrt{\Gamma_{1 r}} \sqrt{\Gamma_{3 r}} b}{a^{2}+b^{2}} \\
K_{22}=-\frac{1}{2}(N-i M) & M=\sum_{r=1}^{N R} \frac{\Gamma_{2 r} a}{a^{2}+b^{2}} & N=\sum_{r=1}^{N R} \frac{\Gamma_{2 r} b}{a^{2}+b^{2}} \\
K_{33}=-\frac{1}{2}(P-i O) & O=\sum_{r=1}^{N R} \frac{\Gamma_{3 r} a}{a^{2}+b^{2}} \\
K_{23}=-\frac{1}{2}(H-i G) & G=\sum_{r=1}^{N R} \frac{\sqrt{\Gamma_{2 r}} \sqrt{\Gamma_{3 r}} a}{a^{2}+b^{2}} & H=\sum_{r=1}^{N R} \frac{\sqrt{\Gamma_{2 r}} \sqrt{\Gamma_{3 r}} b}{a^{2}+b^{2}} \\
a_{3 r}^{2}+b^{2}
\end{array}
$$

\footnotetext{
${ }^{\dagger}$ Note that $a=E_{r}-E, b=\Gamma_{\gamma} / 2$.
} 
An equation for $\rho_{11}$ is obtained by substituting the expressions for $A_{11}, A_{12}$ and $A_{13}$ into Eq. (16). Using the expressions for the elements of the $K$ matrix in Table 1 , the equation for $\rho_{11}$ is expressed as follows:

$$
\rho_{11}=\frac{\alpha(2 \psi+\alpha)+\gamma(2 \chi+\gamma)}{(2 \psi+\alpha)^{2}+(2 \chi+\gamma)^{2}}+i \frac{\alpha(2 \chi+\gamma)-\gamma(2 \psi+\alpha)}{(2 \psi+\alpha)^{2}+(2 \chi+\gamma)^{2}}
$$

where

$$
\begin{aligned}
& \alpha=\left(B^{\prime} x-A^{\prime} y\right)-\left(B^{\prime} u-A^{\prime} v\right)+2 w-q-s, \\
& \gamma=\left(B^{\prime} y+A^{\prime} x\right)-\left(B^{\prime} v-A^{\prime} u\right)+2 z-r-t, \\
& \psi=x-u, \\
& \chi=y-v, \\
& x=(2+N)(2+P)-O M, \\
& y=O(2+N)+M(2+P), \\
& w=D(F H-E G)-C(F G+E H), \\
& z=D(F G+E H)+C(F H-E G), \\
& q=(2+P)\left(D^{2}-C^{2}\right)-2 C D O, \\
& r=2 C D(2+P)+O\left(D^{2}-C^{2}\right), \\
& s=(2+N)\left(F^{2}-E^{2}\right)-2 E F M, \\
& t=2 E F(2+N)+M\left(F^{2}-E^{2}\right), \\
& u=\left(H^{2}-G^{2}\right) \text {, } \\
& v=2 G H .
\end{aligned}
$$


Using the expression for $\rho_{11}$ in Eq. (23), an equation can be developed for $\left|\rho_{11}\right|^{2}=\rho_{11}\left(\rho_{11}\right)^{*}$ :

$$
\left|\rho_{11}\right|^{2}=\frac{[\alpha(2 \psi+\alpha)+\gamma(2 \chi+\gamma)]^{2}+[\alpha(2 \chi+\gamma)-\gamma(2 \psi+\alpha)]^{2}}{\left[(2 \psi+\alpha)^{2}+(2 \chi+\gamma)^{2}\right]^{2}} .
$$

An equation for the quantity $R e \rho_{11}-\left|\rho_{11}\right|^{2}$ is obtained by substituting Eqs. (23) and (38) into Eq. (14). After simplification, the following expression is obtained for $\operatorname{Re} \rho_{11}-\left|\rho_{11}\right|^{2}$ :

$$
R e \rho_{11}-\left|\rho_{11}\right|^{2}=\frac{2(\psi \alpha+\chi \gamma)}{(2 \psi+\alpha)^{2}+(2 \chi+\gamma)^{2}} .
$$

Using Eq. (39), the absorption cross section is obtained as follows:

$$
\sigma_{a}(E)=\frac{4 \pi}{k^{2}} \sum_{J} g_{J} \frac{2(\psi \alpha+\chi \gamma)}{(2 \psi+\alpha)^{2}+(2 \chi+\gamma)^{2}} .
$$

With regard to the capture cross section, the quantity that is defined by Eq. (15) can be evaluated by obtaining expressions for $\left|\rho_{12}\right|^{2}$ and $\left|\rho_{13}\right|^{2}$. An expression for $\rho_{12}$ is obtained by substituting Eqs. (19) through (21) into Eq. (17). After the substitution and algebraic simplifications, the following expression is obtained for $\rho_{12}$ :

$$
\rho_{12}=\frac{-[f(2 \psi+\alpha)-g(2 \chi+\gamma)]}{(2 \psi+\alpha)^{2}+(2 \chi+\gamma)^{2}}-i \frac{[f(2 \chi+\gamma)+g(2 \psi+\alpha)]}{(2 \psi+\alpha)^{2}+(2 \chi+\gamma)^{2}},
$$


where

$$
\begin{gathered}
f=\chi C-\psi D+M \lambda_{2}-N \lambda_{1}+G \lambda_{4}-H \lambda_{3}, \\
g=\psi C+\chi D+M \lambda_{1}+N \lambda_{2}+G \lambda_{3}+H \lambda_{4}, \\
\lambda_{1}=(F H-E G)-[D(2+P)-C O], \\
\lambda_{2}=(F G+E H)-[D O+C(2+P)], \\
\lambda_{3}=(D H-C G)-[F(2+N)-E M], \\
\lambda_{4}=(D G+C H)-[F M+E(2+N)] .
\end{gathered}
$$

Using the expression for $\rho_{12}$ in Eq. (41), an equation can be developed for $\left|\rho_{12}\right|^{2}$ :

$$
\left|\rho_{12}\right|^{2}=\frac{[f(2 \psi+\alpha)-g(2 \chi+\gamma)]^{2}+[f(2 \chi+\gamma)+g(2 \psi+\alpha)]^{2}}{(2 \psi+\alpha)^{2}+(2 \chi+\gamma)^{2}} .
$$

An expression for $\rho_{13}$ is obtained by substituting Eqs. (19) through (21) into Eq. (18). After the substitution and algebraic simplifications, the following expression is obtained for $\rho_{13}$ :

$$
\rho_{13}=\frac{-[h(2 \psi+\alpha)-k(2 \chi+\gamma)]}{(2 \psi+\alpha)^{2}+(2 \chi+\gamma)^{2}}-i \frac{[h(2 \chi+\gamma)+k(2 \psi+\alpha)]}{(2 \psi+\alpha)^{2}+(2 \chi+\gamma)^{2}}
$$

where

$$
\begin{aligned}
& h=\chi E-\psi F+G \lambda_{2}-H \lambda_{1}+O \lambda_{4}-P \lambda_{3}, \\
& k=\psi E+\chi F+G \lambda_{1}+H \lambda_{2}+O \lambda_{3}+P \lambda_{4} .
\end{aligned}
$$

After multiplying $\rho_{13}$ by the complex conjugate, the following expression is obtained for $\left|\rho_{13}\right|^{2}$ : 


$$
\left|\rho_{13}\right|^{2}=\frac{[h(2 \psi+\alpha)-k(2 \chi+\gamma)]^{2}+[h(2 \chi+\gamma)+k(2 \psi+\alpha)]^{2}}{(2 \psi+\alpha)^{2}+(2 \chi+\gamma)^{2}} .
$$

An equation for the quantity $R e \rho_{11}-\left|\rho_{11}\right|^{2}-\left|\rho_{12}\right|^{2}-\left|\rho_{13}\right|^{2}$ is obtained by substituting Eqs. (39), (48) and (52) into Eq. (15):

$$
\operatorname{Re} \rho_{11}-\left|\rho_{11}\right|^{2}-\left|\rho_{12}\right|^{2}-\left|\rho_{13}\right|^{2}=\frac{2(\psi \alpha+\chi \gamma)-\left(f^{2}+g^{2}+h^{2}+k^{2}\right)}{(2 \psi+\alpha)^{2}+(2 \chi+\gamma)^{2}} .
$$

Using Eq. (53), the capture cross section can be expressed as follows:

$$
\sigma_{\gamma}(E)=\frac{4 \pi}{k^{2}} \sum_{J} g_{J} \frac{2(\psi \alpha+\chi \gamma)-\left(f^{2}+g^{2}+h^{2}+k^{2}\right)}{(2 \psi+\alpha)^{2}+(2 \chi+\gamma)^{2}} .
$$

In an effort to demonstrate the numerical aspects of the RM formalism, absorption and capture cross-sections were calculated using Eqs. (12) and (13), respectively. For the purposes of discussion, Eqs. (12) and (13) will be referred to as the "original" RM formulation for the absorption and capture cross sections. In modern FORTRAN, the evaluation of Eqs. (12) and (13) can be performed using double precision complex variables, and the subsequent complex arithmetic is handled by the compiler. For comparison purposes, Eqs. (40) and (54) were also used to calculate absorption and capture cross sections as a function of energy, respectively. The form of the absorption and capture cross sections as defined by Eqs. (40) and (54) will be referred to as the "revised" RM formulation for absorption and capture. The cross-section calculations were performed with the AMPX module POLIDENT (Dunn and Greene, 2000) that is used to generate continuous energy cross sections from ENDF data at ORNL. All the calculations were performed on a DEC Alpha AS 500/500 workstation.

The capture cross section as a function of energy was calculated for ENDF/B- $6{ }^{28} \mathrm{Si}$, and the calculated results for the $298.7 \mathrm{keV} d$-wave capture resonance are presented in Fig. 1. Since ${ }^{28} \mathrm{Si}$ is a nonfissionable isotope, the fission channels are empty and the equations for absorption and capture are equivalent. For comparison, the capture cross section for ${ }^{28} \mathrm{Si}$ was calculated as a function of energy using Eq. (54), and the results are also presented in Fig. 1. Although both forms of the capture cross section provide the general structure of the resonance, the original RM formulation leads to a nonphysical oscillatory behavior in the cross section on the wings of the capture resonance. In contrast, the revised RM formulation exhibits a smooth structure over the resonance. From a practical standpoint, the oscillations provided by the original formulation are within the measurement uncertainty of the actual cross-section value; however, the oscillatory behavior of the original RM formulation does provide numerical convergence problems for an 
adaptive energy-meshing scheme that may be used to determine the appropriate energy-grid structure for the resonance. Consequently, using the form of the capture cross section as presented by Eq. (13) is not desirable for generating cross sections in conjunction with an adaptive energy-meshing scheme as used by POLIDENT. Implementation of the revised RM formulation leads to a 1 to $2 \%$ increase in CPU time relative to the original RM formulation. Therefore, the revised RM formulation is relatively inexpensive as compared with the original RM formulation. Calculations were also performed for ${ }^{28} \mathrm{Si}$ capture using the Multilevel R-matrix code SAMMY (Larson, 2000), and no numerical problems were observed with the SAMMY formulation of the RM approximation.

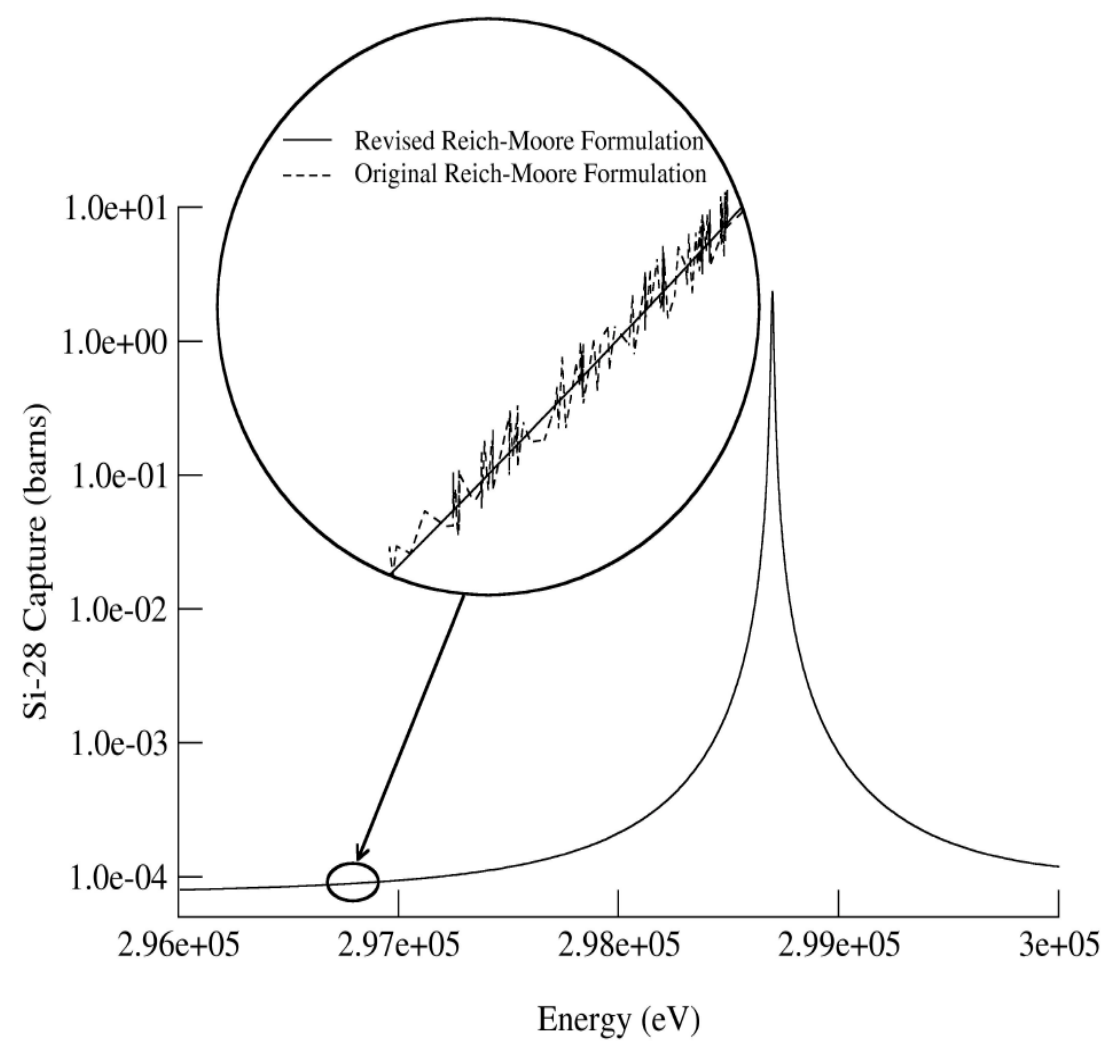

Fig. 1 Reich-Moore representation of the ENDF/B- $6{ }^{28} \mathrm{Si} 298.7 \mathrm{keV}$ capture resonance. 


\subsection{Elastic Scattering}

The Reich-Moore expression for the elastic scattering cross section is provided in Eq. (9). If the single- or double-precision FORTRAN complex functions are used to evaluate $\sigma_{\mathrm{el}}(E)$, a numerical instability can occur in Eq. (9) under the following condition:

$$
\sin ^{2} \phi_{1} \approx-2 \sin ^{2} \phi_{1} \operatorname{Re} \rho_{11}+\sin \left(2 \phi_{1}\right) \operatorname{Im} \rho_{11}+\left|\rho_{11}\right|^{2} .
$$

To circumvent the instability problem, an expression can be developed for the quantity within braces in Eq. (9). In particular, the following equation can be used to express the quantity within braces in Eq. (9):

$$
q t y=\sin ^{2} \phi_{1}-2 \sin ^{2} \phi_{1} \operatorname{Re} \rho_{11}+\sin \left(2 \phi_{1}\right) \operatorname{Im} \rho_{11}+\left|\rho_{11}\right|^{2} .
$$

Using the expressions for $\rho_{11}$ and $\left|\rho_{11}\right|^{2}$ from Eqs. (23) and (38), respectively, an equation can be developed for qty. After substituting Eqs. (23) and (38) into Eq. (56) and simplifying the expression, the following equation is obtained for qty:

$$
q t y=\frac{2\left[2 \sin ^{2} \phi_{1}\left(\psi^{2}+\chi^{2}\right)+\sin 2 \phi(\alpha \chi-\gamma \psi)\right]+\left(\alpha^{2}+\gamma^{2}\right)\left(1-\sin ^{2} \phi_{1}\right)}{(2 \psi+\alpha)^{2}+(2 \chi+\gamma)^{2}} .
$$

Following a similar procedure for the capture cross section, the elastic scattering cross section was calculated using Eq. (9), and the form of the elastic scattering cross section in Eq. (9) will be referred to as the original RM formulation. For comparison, the quantity in Eq. (57) is used to evaluated the quantity within braces in Eq. (9). The form of the elastic scattering cross section that uses the expression defined by Eq. (57) will be referred to as the revised RM formulation.

Using POLIDENT, the elastic scattering cross section as a function of energy was calculated for ENDF/B- $6{ }^{60} \mathrm{Ni}$, and the results for the elastic scattering reaction between $4 \mathrm{keV}$ and $5.4 \mathrm{keV}$ are provided in Fig. 2. The dip in the ${ }^{60} \mathrm{Ni}$ scattering cross section occurs at $4.91 \mathrm{keV}$ between two $p$-wave resonances at $2.253 \mathrm{keV}$ and $5.5301 \mathrm{keV}$. The original RM representation for the cross section was generated by using double precision complex FORTRAN variables to evaluate the matrix $\rho_{11}$ and subsequently Eq. (9). As shown in Fig. 2, the original RM formulation breaks down between $4.8 \mathrm{keV}$ and $4.9 \mathrm{keV}$ as the scattering cross section approaches $1 \times 10^{-4}$ barns before the minimum value of $7.74 \times 10^{-5}$ barns. In contrast, the revised RM 
formulation provides a smooth structure throughout the dip in the ${ }^{60} \mathrm{Ni}$ elastic scattering cross section. As noted for the ${ }^{28} \mathrm{Si}$ capture calculation, the oscillations in the elastic scattering cross section are within the experimental uncertainty of the actual cross-section value; however, the oscillatory behavior provides numerical convergence problems for an adaptive energy-meshing scheme. Therefore, the revised RM formulation is needed to avoid the numerical instability that is shown in Fig. 2 for the original RM formulation. In terms of CPU cost, implementation of the revised RM formulation relative to the original formulation is the same as observed for the absorption and capture cross sections (i.e., 1 to $2 \%$ increase in CPU time for the revised RM formulation). Note that SAMMY was also used to evaluate the ${ }^{60} \mathrm{Ni}$ elastic scattering cross section, and no numerical problems were observed with the SAMMY formulation of the RM approximation.

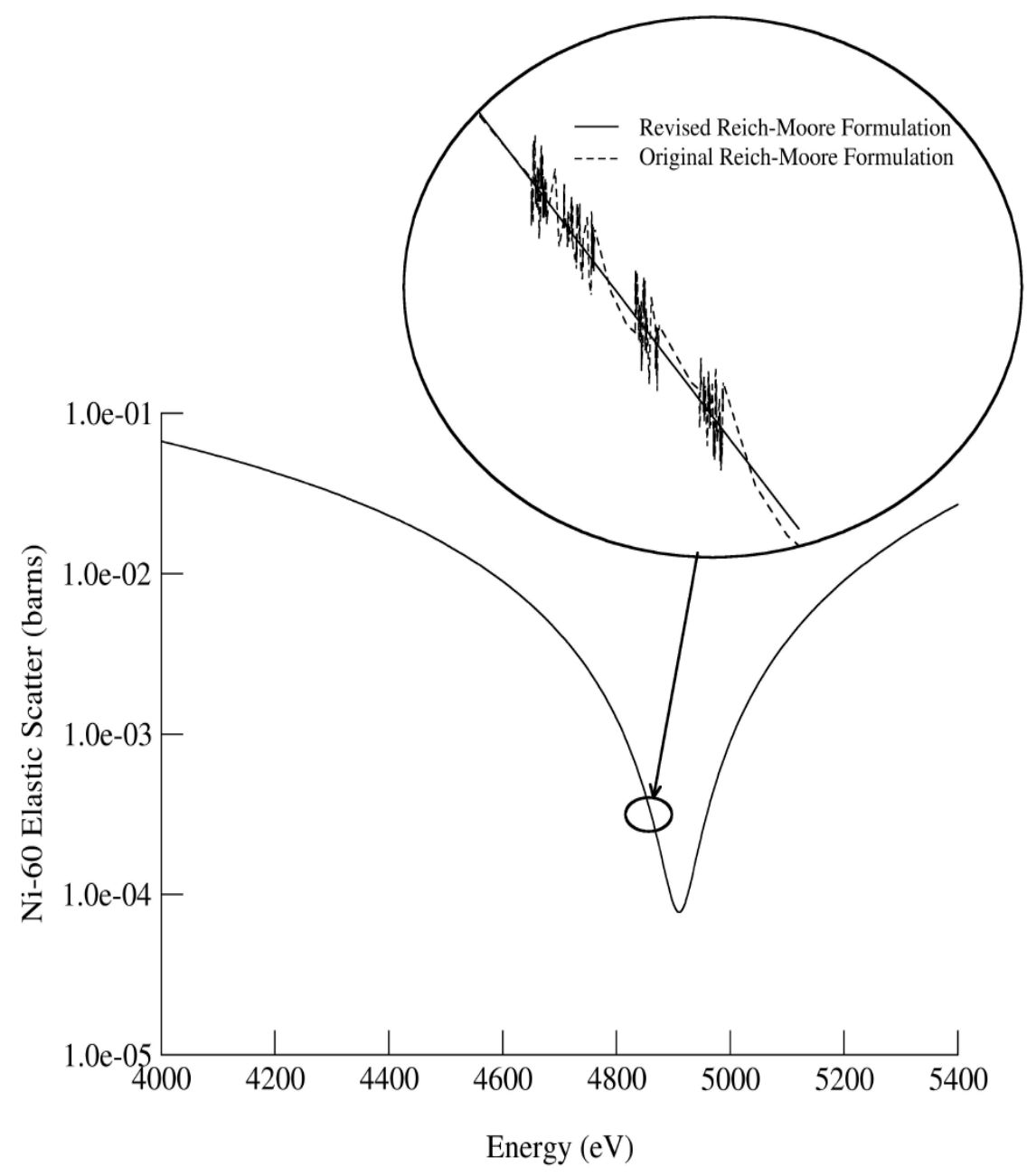

Fig. 2 Reich-Moore representation of the ENDF/B- $6{ }^{60} \mathrm{Ni}$ elastic scattering cross section. 


\section{CONCLUSIONS}

The Reich-Moore (RM) cross-section representation, which is an approximation to general R-matrix theory, is used extensively in many isotope/nuclide evaluations to describe neutron cross sections for the resolved-resonance region. The RM equations involve the evaluation of complex matrices that are a function of the resonance energy and resonance widths. With modern FORTRAN, double precision complex variables can be used to evaluate the the RM equations. As a result, the burden of complex arithmetic is placed on the FORTRAN compiler. Using FORTRAN complex arithmetic is adequate for evaluating the RM equations for many isotopes of interest; however, numerical instabilities with FORTRAN complex operations can occur for isotopes that are characterized by very small aborption or capture cross-section values (e.g., $\sim 10^{-5}$ ) with a detailed resonance structure. The numerical problems occur when the complex operations involve the subtraction of two small numbers that are approximately equal. Moreover, the numerical instabilities occur during capture or absorption cross-section calculations on an very dense energy grid. Similar numerical instabilities can occur in the elastic scattering cross-section calculation. Because of the computational problems associated with the FORTRAN double precision complex operiations in the RM formulation, alternative expressions are needed to accurately calculate the absorption, capture and elastic scattering cross-section values.

This paper presents the conventional RM formulae and identifies the implementation problems that can lead to numerical instabilities in cross-section calculations for the absorption, capture and elastic scattering cross sections. As an alternative, detailed expressions for the $3 \times 3 \mathrm{RM}$ equations that can be used to avoid numerical problems in the cross-section calculation have been developed for calculations in the resolved-resonance region.

In an effort to test the revised RM equations, cross sections were calculated for ENDF/B$6{ }^{28} \mathrm{Si}$ and ${ }^{60} \mathrm{Ni}$. All the calculations were performed on a DEC Alpha AS 500/500 workstation using the AMPX module POLIDENT that is used to generate continuous energy cross sections from ENDF data. For ${ }^{28} \mathrm{Si}$, capture cross sections were calculated for the $298.7 \mathrm{keV} d$-wave resonance using FORTRAN double precision complex operations to evaluate the RM equations (i.e., original formulation). For comparison, the ${ }^{28} \mathrm{Si}$ capture resonance was also calculated using the alternative or revised RM equations that were developed for this paper. With the original RM formulation, nonphysical oscillations were observed on the wings of the ${ }^{28} \mathrm{Si}$ capture resonance; however, the numerial problems were not observed with the revised RM equations. Regarding ${ }^{60} \mathrm{Ni}$, the original RM formulation was used to calculate the elastic scattering cross section as a function of energy. At $4.91 \mathrm{keV},{ }^{60} \mathrm{Ni}$ has a sharp dip in the scattering cross section, and using the FORTRAN double precision complex operations to evaluate the RM equations leads to nonphysical oscillations in the elastic scattering cross section between 4.8 and $4.9 \mathrm{keV}$. With the revised RM formulation, the numerical problems are not observed in the elastic scattering calculation. In terms of additional computing cost for the absorption, capture and elastic scattering, the revised RM formulation only leads to a 1 to $2 \%$ increase in CPU time relative to 
the original RM formulation. Therefore, the revised RM formulation is relatively efficient and can be used to accurately calculate cross sections in the resolved-resonance region.

\section{NOMENCLATURE}

$\delta \quad$ Kronecker delta function

$\Gamma \quad$ partial resonance width

$\phi \quad$ angular momentum hard-sphere phase shift

$\pi \quad$ pi

E energy (eV)

ENDF Evaluated Nuclear Data File

$g \quad$ statistical spin factor

$k \quad$ neutron-wave number at energy $E$

I identity matrix

Im imaginary component of a complex quantity

NR number of resonances

ORNL Oak Ridge National Laboratory

$R e \quad$ real component of a complex quantity

RM Reich-Moore

$U$ collision or scattering matrix for neutron-induced reactions

\section{Subscripts}

$\ell \quad$ angular momentum index

$\gamma \quad$ capture cross section or neutron capture width

$a$ absorption cross section

$c \quad$ exit channel

el elastic scattering cross section

$J \quad$ spin of the resonance

$n$ entrance channel for neutron-induced reactions or neutron width

$r$ resonance index

$t \quad$ total cross section

\section{Superscripts}

$J \quad$ spin of the resonance 


\section{ACKNOWLEDGMENTS}

The author wishes to acknowledge the U.S. Nuclear Regulatory Commision for providing the funding for this work. In addition, gratitude is expressed to N. M. Larson for testing the RM formulation in SAMMY by calculating cross sections for ${ }^{28} \mathrm{Si}$ and ${ }^{60} \mathrm{Ni}$.

\section{REFERENCES}

ENDF/102 Data Formats and Procedures for the Evaluated Nuclear Data File ENDF-6, BNLNCS-44945, Rev. 10/91 (ENDF/B-VI), Brookhaven National Laboratory, October 1991.

Dunn, M. E., and N. M. Greene, POLIDENT: A Module for Generating Continuous-Energy Cross Sections from ENDF Resonance Data, NUREG/CR-6694 (ORNL/TM-2000/035), U.S. Nuclear Regulatory Commission, Oak Ridge National Laboratory, December 2000.

Larson, N. M., "Cross Section Parameterization in the Resolved Resonance Region," invited paper presented October 12-16, 1992, at Brookhaven National Laboratory and published in International Symposium on Nuclear Data Evaluation Methodology, Ed., Charles L. Dunford, pp. 285-294, 1993.

Larson, N. M., Updated Users' Guide for SAMMY Multilevel R-matrix Fits to Neutron Data using Bayes' Equation, ORNL/TM-9179/R5 (ENDF-364), UT-Battelle, LLC, Oak Ridge National Laboratory, November 2000. 